See discussions, stats, and author profiles for this publication at: https://www.researchgate.net/publication/307631934

\title{
The mechanics of decompressive craniectomy: Personalized simulations
}

Article in Computer Methods in Applied Mechanics and Engineering • August 2016 DOI: 10.1016/j.cma.2016.08.011

\section{CITATIONS}

12

5 authors, including:

Celia A. M. Butler

11 PUBLICATIONS 88 CITATIONS

SEE PROFILE

Alain Goriely

University of Oxford

274 PUBLICATIONS 7,281 CITATIONS

SEE PROFILE

Some of the authors of this publication are also working on these related projects:

Perovskite Photovoltaics View project

Project The Mathematics and Mechanics of Biological Growth View project
26. Philippe Young

University of Exeter

264 PUBLICATIONS 1,511 CITATIONS

SEE PROFILE 


\title{
The mechanics of decompressive craniectomy: Personalized simulations
}

\author{
J. Weickenmeier ${ }^{\mathrm{a}}$, C. Butler ${ }^{\mathrm{b}}$, P.G. Young ${ }^{\mathrm{c}}$, A. Goriely ${ }^{\mathrm{d}}$, E. Kuhl ${ }^{\mathrm{a}, \mathrm{e}, *}$ \\ ${ }^{a}$ Department of Mechanical Engineering, Stanford University, Stanford, CA 94305, USA \\ ${ }^{b}$ Simpleware Ltd., Bradninch Hall, Castle Street, Exeter EX4 3PL, UK \\ ${ }^{c}$ College of Engineering, Mathematics and Physical Sciences, University of Exeter, Exeter, Devon, UK \\ ${ }^{d}$ Mathematical Institute, University of Oxford, Oxford, UK \\ ${ }^{e}$ Department of Bioengineering, Stanford University, Stanford, CA 94305, USA
}

\begin{abstract}
Decompressive craniectomy is a traditional but controversial surgical procedure that removes part of the skull to allow an injured and swollen brain to expand outward. Recent studies suggest that mechanical strain is associated with its undesired, high failure rates. However, the precise strain fields induced by the craniectomy are unknown. Here we create a personalized craniectomy model from magnetic resonance images to quantify the strains during a decompressive craniectomy using finite element analysis. We swell selected regions of the brain and remove part of the skull to allow the brain to bulge outward and release the intracranical swelling pressure. Our simulations reveal three potential failure mechanisms associated with the procedure: axonal stretch in the center of the bulge, axonal compression at the edge of the craniectomy, and axonal shear around the opening. Strikingly, for a swelling of only $10 \%$, axonal strain, compression, and shear reach local maxima of up to $30 \%$, and exceed the reported functional and morphological damage thresholds of $18 \%$ and $21 \%$. Our simulations suggest that a collateral craniectomy with the skull opening at the side of swelling is less invasive than a contralateral craniectomy with the skull opening at the opposite side: It induces less deformation, less rotation, smaller strains, and a markedly smaller midline shift. Our computational craniectomy model can help quantify brain deformation, tissue strain, axonal stretch, and shear with the goal to identify high-risk regions for brain damage on a personalized basis. While computational modeling is beyond clinical practice in neurosurgery today, simulations of neurosurgical procedures have the potential to rationalize surgical process parameters including timing, location, and size, and provide standardized guidelines for clinical decision making and neurosurgical planning.
\end{abstract}

Keywords: Neuromechanics; neurosurgery; finite element analysis; decompressive craniectomy; hemicraniectomy

\section{Motivation}

Decompressive craniectomy is a life-saving neurosurgical procedure in which part of the skull is removed to provide additional space for a swollen brain [32]. The surgical opening of the skull has been performed for more than a century to reduce an elevated intracranial pressure in patients with traumatic brain injury, subarachnoid hemorrhage, and ischemic stroke [31]. Yet, because of its invasive nature, the procedure remains controversial and ethically questionable [27]. While recent clinical trials suggest that decompressive craniectomy improves short-term pressure management and survival compared to pharmaceutical treatment alone, survivors of the procedure often experience moderate to severe long-term disabilities [8, 26]. These disabilities raise ethical concerns about appropriate selection criteria for patients who could benefit from the procedure [45]. Current guidelines suggest to perform a craniectomy when the intracranial pressure is greater than $20 \mathrm{mmHg}$ for more than 30 minutes [28]. A chronically elevated intracranial pressure can be devastating or, in the worst case, fatal; it causes a reduction in cerebral perfusion pressure, cerebral blood flow, and oxygenation [32].

Decompressive craniectomy is often performed as a treatment of last resort to manage elevated pressure levels when medical management alone has proven unsuccessful. The procedure is based on removing large portions of the skull, opening the dura, the protective membrane that surrounds the brain, and allowing the brain to expand outward. The removed bone flap is typically preserved and re-implanted within the following weeks, once the swelling has been fully resorbed [46]. Despite a marked increase in popularity, decompressive craniectomy remains a compromise between maximizing control of the intracranial pressure and minimizing side effects to the surrounding tissue [50]. To date, the precise criteria when to perform a decompressive craniectomy,

\footnotetext{
${ }^{*}$ Corresponding author

Email address: ekuhl@stanford.edu (E. Kuhl)
} 
the optimal timing of treatment, the optimal location and size of the skull opening, and the long-term functional outcome remain unclear.

The location of the skull opening is a matter of ongoing debate. Decompressive craniectomy can be either unilateral with an opening on the left or right hemisphere, or bilateral with a bifrontal opening across both hemispheres [32]. Unilateral decompressive craniectomy, or hemicraniectomy, is by far the most common procedure [41]. It is recommended in patients with unilateral hemispheric edema, a condition that can be diagnosed radiologically via a midline shift to the contralateral side [27]. Under these conditions, it is common practice to perform a collateral craniectomy and open the skull at the side of swelling. Surprisingly, recent computational studies suggest that tissue strains can be reduced by performing a contralateral craniectomy at the opposite, non-injured side of the brain [23]. Yet, the mechanisms that reduce tissue strains during contralateral skull opening are far from completely understood.

The size of the skull opening is another clinical parameter of critical importance, especially in unilateral craniectomy where the surgical space is anatomically limited. Studies report a direct correlation between the amount of bone removal and the reduction in intracranial pressure, which was found to be less than $40 \%$ when removing $8,000 \mathrm{~mm}^{3}$ of bone and greater than $80 \%$ when removing $12,000 \mathrm{~mm}^{3}$ [28]. Too large openings are associated with high rates of infection, complications during wound healing, challenges with cranial reconstruction, and a higher risk to develop hydrocephalus [32]. Too small openings increase the risk of external cerebral herniation, an outward squeezing of the brain, which results in compression of the bridging veins, venous infarction, and increased edema at the bone margins [46]. From a mechanical point of view, smaller openings naturally induce larger strains in the outward bulging tissue and increase the risk of tissue damage [22]. As a result, guidelines recommend a minimum opening diameter on the order of $12 \mathrm{~cm}$ [47]. Yet, this recommendation seems to be based on surgical experience rather than on a sound quantitative analysis.

Mathematical modeling is increasingly recognized as a promising strategy to predict the deformation field during decompressive craniectomy, the dimensions of the outward bulging tissue, the resulting strain field, and the regions at risk for local tissue damage [14]. The complexity of existing models ranges from analytical models for idealized axisymmetric hyperelastic materials interpreted as an inverted punch problem [20] to finite element models for idealized axisymmetric poro-visco-elastic materials calibrated with gelatin hydrogel experiments [12]. Gelatin models are currently gaining popularity as precisely tunable surrogates of the human brain and provide valuable qualitative insight into brain deformation in neurosurgical training and pre-operative planning [40]. At the same time, as our knowledge of brain mechanics increases, our computational models become more sophisticated. Since the first computational craniectomy model was presented less than a decade ago [16], significant progress has been made towards making the model anatomically more realistic [30]. To date, however, the most frequent application of computational brain models is high impact loading in traumatic brain injury [10], and the use of computational models in surgical simulations remains the exception.

Computational modeling allows us to virtually explore potential failure mechanisms, not only in response to high impact loading, but also in response to surgical procedures. Elevated axonal strain has been hypothesized as a possible failure mechanism and tested in controlled single axon [48] and nerve tissue [2] experiments in vitro. These findings have inspired microscopically motivated, anisotropic brain tissue models to quantify the axonal strains and von Mises stresses around a circular inclusion [7]. These two metrics are associated with the failure mechanisms of axonal stretch and axonal shear [10]. The definition of appropriate stressand strain-based failure criteria and damage thresholds is still hotly debated with suggested use of maximum principal values, von Mises values, or their projections normal and tangential to the axonal direction [51]. The objective of this study is to compare different strain-based damage criteria and to identify regions of maximum principal strain, radial stretch, and tangential stretch in response to a unilateral decompressive craniectomy using a personalized, anatomically detailed model of the human brain.

\section{Methods}

\subsection{Constitutive model}

We begin by briefly summarizing the constitutive model for brain tissue based on a classical hyperelastic constitutive formulation [24]. On time scales on the order of milliseconds or seconds characteristic for traumatic brain injury, the brain behaves poro-visco-elastic and is highly sensitive to the loading rate [15, 34]. On the time scales on the order of minutes or hours characteristic for a surgical procedure, it seems reasonable to approximate the brain as purely hyperelastic [5, 6]. Motivated by the literature, we follow the recommendation to model brain tissue as an isotropic material [6], but interpret the resulting strain and stress fields in view of their projections onto pronounced axonal directions [51]. To characterize the behavior of the brain at finite deformations, we introduce the deformation gradient $\boldsymbol{F}$ as the gradient of the nonlinear deformation field $\boldsymbol{\varphi}$ with respect to the material coordinates $\boldsymbol{X}$ in the reference configuration. To account for the nearly incompressible behavior of brain tissue, we decompose the deformation gradient $\boldsymbol{F}$ into a volumetric contribution characterized through the Jacobian $J$ and an isochoric contribution $\overline{\boldsymbol{F}}$,

$$
\boldsymbol{F}=\nabla_{X} \boldsymbol{\varphi}=J^{1 / 3} \overline{\boldsymbol{F}} \quad \text { with } \quad J=\operatorname{det}(\boldsymbol{F}) \quad \text { and } \quad \overline{\boldsymbol{F}}=J^{-1 / 3} \boldsymbol{F} .
$$

As a characteristic deformation measure, we introduce the left Cauchy Green deformation tensor $\boldsymbol{b}$, which obeys a similar decomposition into a volumetric contribution in terms of the Jacobian $J$ and an isochoric contribution $\bar{b}$,

$$
\boldsymbol{b}=\boldsymbol{F} \cdot \boldsymbol{F}^{\mathrm{t}}=J^{2 / 3} \overline{\boldsymbol{b}} \quad \text { with } \quad J^{2 / 3}=\operatorname{det}^{2 / 3}(\boldsymbol{F}) \quad \text { and } \quad \overline{\boldsymbol{b}}=\overline{\boldsymbol{F}} \cdot \overline{\boldsymbol{F}}^{\mathrm{t}} .
$$


We can then introduce the isochoric first and second invariants, $\bar{I}_{1}$ and $\bar{I}_{2}$, either in terms of the isochoric left Cauchy Green deformation tensor $\overline{\boldsymbol{b}}$ or in terms of the isochoric principal stretches $\bar{\lambda}_{1}, \bar{\lambda}_{2}$, and $\bar{\lambda}_{3}$, recalling that $\bar{I}_{3}=\bar{J}^{2}=1$,

$$
\bar{I}_{1}=\operatorname{tr}(\overline{\boldsymbol{b}})=\bar{\lambda}_{1}^{2}+\bar{\lambda}_{2}^{2}+\bar{\lambda}_{3}^{2} \quad \text { and } \quad \bar{I}_{2}=\frac{1}{2}\left[\operatorname{tr}^{2}(\overline{\boldsymbol{b}})-\operatorname{tr}\left(\overline{\boldsymbol{b}}^{2}\right)\right]=\bar{\lambda}_{1}^{2} \bar{\lambda}_{2}^{2}+\bar{\lambda}_{2}^{2} \bar{\lambda}_{3}^{2}+\bar{\lambda}_{3}^{2} \bar{\lambda}_{1}^{2}
$$

Many common constitutive models for brain tissue are special cases of the general Ogden model [39],

$$
\psi=\sum_{\mathrm{i}=1}^{N} \frac{c_{\mathrm{i}}}{\alpha_{i}}\left[\bar{\lambda}_{1}^{\alpha_{\mathrm{i}}}+\bar{\lambda}_{2}^{\alpha_{\mathrm{i}}}+\bar{\lambda}_{3}^{\alpha_{\mathrm{i}}}-3\right]+U(J),
$$

where $c_{\mathrm{i}}$ and $\alpha_{\mathrm{i}}$ are material-specific parameters and the function $U(J)$ controls the volumetric response. For the special case of $N=2$ with $\alpha_{1}=2$ and $\alpha_{2}=-2$, the Ogden model simplifies to the popular Mooney-Rivlin model [38, 42],

$$
\psi=\frac{1}{2} c_{1}\left[\bar{\lambda}_{1}^{2}+\bar{\lambda}_{2}^{2}+\bar{\lambda}_{3}^{2}-3\right]+\frac{1}{2} c_{2}\left[\bar{\lambda}_{1}^{-2}+\bar{\lambda}_{2}^{-2}+\bar{\lambda}_{3}^{-2}-3\right]+U(J)
$$

which we can reformulate in terms of the isochoric invariants (3),

$$
\psi=\frac{1}{2} c_{1}\left[\bar{I}_{1}-3\right]+\frac{1}{2} c_{2}\left[\bar{I}_{2}-3\right]+U(J) .
$$

The two Mooney-Rivlin parameters $c_{1}$ and $c_{2}$ are related to the shear modulus $\mu$ as $2\left[c_{1}+c_{2}\right]=\mu$. The Piola stress of the Mooney-Rivlin model follows from the Clausius Duhem inequality,

$$
\boldsymbol{P}=\frac{\partial \psi}{\partial \boldsymbol{F}}=\frac{1}{J^{2 / 3}}\left[c_{1}+\bar{I}_{1} c_{2}\right] \boldsymbol{F}-\frac{1}{J^{4 / 3}} c_{2} \boldsymbol{F} \cdot \boldsymbol{F}^{\mathrm{t}} \cdot \boldsymbol{F}-\frac{1}{3}\left[\bar{I}_{1} c_{1}+2 \bar{I}_{2} c_{2}\right] \boldsymbol{F}^{-\mathrm{t}}+J \frac{\partial U}{\partial J} \boldsymbol{F}^{-\mathrm{t}},
$$

and the Kirchhoff stress, $\boldsymbol{\tau}=\boldsymbol{P} \cdot \boldsymbol{F}^{\mathrm{t}}$, is the partial push-forward of the Piola stress,

$$
\boldsymbol{\tau}=2 \frac{\partial \psi}{\partial \boldsymbol{b}} \cdot \boldsymbol{b}=\frac{1}{J^{2 / 3}}\left[c_{1}+\bar{I}_{1} c_{2}\right] \boldsymbol{b}-\frac{1}{J^{4 / 3}} c_{2} \boldsymbol{b}^{2}-\frac{1}{3}\left[\bar{I}_{1} c_{1}+2 \bar{I}_{2} c_{2}\right] \boldsymbol{I}+J \frac{\partial U}{\partial J} \boldsymbol{I} .
$$

In the case of quasi-incompressibility, we could make a volumetric ansatz, $U(J)=\frac{1}{2} \kappa[J-1]^{2}$, and the derivative $\partial U / \partial J=$ $\kappa[J-1]=-p$ would take the interpretation of the pressure $p$. In the case of incompressibility, where $J=1, p$ no longer follows from a constitutive equation; instead, $p$ becomes an arbitrary Lagrange multiplier associated with the incompressibility constraint, $J=1$. Given the incompressible nature of brain tissue, we follow the second approach and use a hybrid element formulation and evaluate the volumetric term using a mixed element approach to enforce the incompressibility constraint [1].

Table 1: Material parameters of the Mooney-Rivlin model in different tissue types.

\begin{tabular}{|l|c|c|}
\hline substructure & parameter $c_{1}$ [kPa] & parameter $c_{2}$ [kPa] \\
\hline \hline cerebral gray matter & 0.28 & 333.0 \\
cerebral white matter & 0.56 & 666.0 \\
cerebellum & 0.28 & 333.0 \\
cerebrospinal fluid & 0.03 & 33.3 \\
\hline
\end{tabular}

We adapt the Mooney-Rivlin parameters $c_{1}=0.28 \mathrm{kPa}$ and $c_{2}=333 \mathrm{kPa}$ reported for brain tissue in the literature [37]. We assume that the cerebral white matter is twice as stiff as the cerebral gray matter and the cerebellum [49]. For simplicity, we model the cerebrospinal fluid as an ultrasoft solid with a ten times lower stiffness than the cerebral gray matter. We assume that the individual tissue types display neither directional [7] nor regional [9] variations, and model them as isotropic [6] and homogeneous [29]. We further assume that all soft tissue as incompressible, and discretize them using hybrid linear tetrahedral C3D4H elements [1].

To model brain swelling, we multiplicatively decompose the total Jacobian $J=J^{\mathrm{e}} J^{\mathrm{s}}$ into an elastic part $J^{\mathrm{e}}$ and a swelling part $J^{s}$. We gradually increase the amount of swelling from $J^{s}=1.0$ to $J^{s}=1.10$ to model a volumetric swelling of $10 \%$. In the case of swelling, we re-parameterize the volumetric term $U(J)$ in the definitions of the free energies (4), (5), and (6), the Piola stress (7), and Kirchhoff stress (8) in terms of the elastic Jacobian $U\left(J^{\mathrm{e}}\right)=U\left(J / J^{\mathrm{s}}\right)$. Within our computational model, we represent volumetric swelling via volumetric thermal expansion [1], and only allow selected regions of the cerebral white matter tissue to swell, while all other substructures remain purely elastic. 


\subsection{Magnetic resonance imaging}

Figure 1 shows representative transverse, sagittal, and coronal slices of an adult female head that form the basis of our model. We acquired these images at the Stanford University Center for Cognitive and Neurobiological Imaging using a 3Tesla scanner (GE MR750, Milwaukee, WI) with a 32-channel radiofrequency receive head coil (Nova Medical, Inc., Wilmington, MA). The collected data set contains 190 slices in the sagittal plane taken at a spacing of $0.9 \mathrm{~mm}$. Each image slice has a matrix representation of $256 \times 256$ pixels with an in-plane resolution of $0.9 \mathrm{~mm} \times 0.9 \mathrm{~mm}$ [44]. From the magnetic resonance images, we create a personalized high-resolution finite element model of the brain. The individual gray scales allow us to generate anatomically detailed and geometrically accurate three-dimensional reconstructions of the individual substructures including the cerebral gray and white matter, the cerebrospinal fluid, the cerebellum, the skin, and the skull.

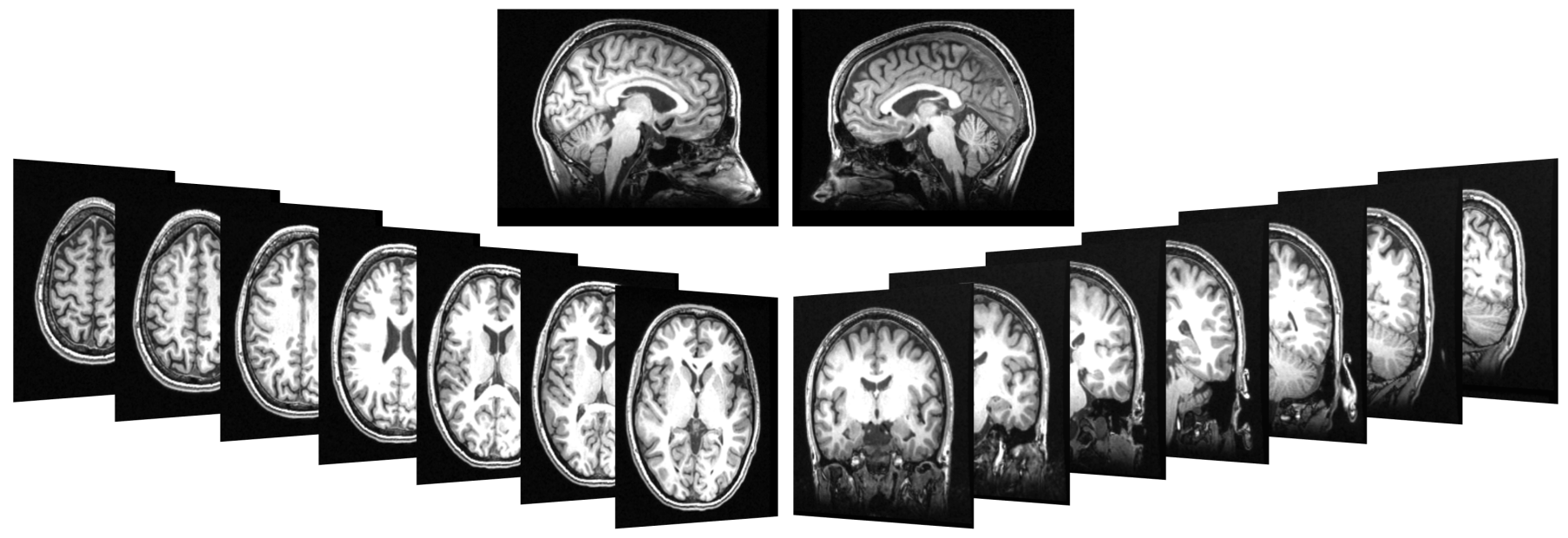

Figure 1: Magnetic resonance images of an adult female brain. The image set contains 190 slices in the sagittal plane taken at a spacing of 0.9 mm. Each image slice has a matrix representation of $256 \times 256$ pixels with an in-plane resolution of $0.9 \mathrm{~mm} \times 0.9 \mathrm{~mm}$. Images were acquired using a 3 Tesla scanner with a 32 -channel radiofrequency receive head coil.

\subsection{Finite element model}

Figure 2 illustrates the results of image processing, segmentation, and model reconstruction using the ScanIP software environment (Simpleware Ltd, Exeter, UK). ScanIP features gray-scale filtering to differentiate between individual tissue layers [52]. This semi-automatic process iteratively produces an optimal segmentation of all relevant substructures [9]. In particular, we segmented a homogenized layer of skin, the skull, and the soft tissues that make up the brain. Our model features a high resolution representation of the brain's gray and white matter tissue. In addition, it contains the cerebellum in the posterior part of the brain and the cerebrospinal fluid which fills all cavities inside the skull. Figure 2 documents multiple representations of the geometric reconstruction, with the skull shown in gray, the skin layer in brown, the cortical gray matter in red, the inner white matter in pink, the cerebellum in green, and the cerebrospinal fluid in beige. The top layer shows the finite element mesh of the model.

In the cerebrum, our model differentiates between cerebral gray and white matter tissue for three mechanically and medically relevant reasons: First, gray and white matter possess clearly distinct microstructures that give rise to a different constitutive response [5, 49]. Second, during neurodevelopment, the interplay between gray and white matter plays a critical role in modulating the characteristic folding pattern of our brain, and our model should be sufficiently general to be applicable in such situations [4]. Third, during most human neuropathologies, alterations affect either the gray or white matter tissue and to properly represent the etiology and disease progression, it is important to represent cerebral gray and white matter as individual substructures.

In the cerebellum, our model simplifies the discretization to reduce model complexity. The human cerebellum is the most folded structure of our brain, mainly because of its unique function during sensory acquisition, motor control, and regulation [35]. The significant folding enables high sensitivity and short signaling pathways, but is complex to account for in the computational model. In view of the complex interaction between the cerebellum and the cerebrospinal fluid as well as the cerebellum and cerebral gray matter we model the cerebellum as a single homogenized material.

Figure 2 shows multiple representations of our finite element model generated using our finite element meshing tool (Simpleware Ltd, Exeter, UK). The full head model consists of 1,275,808 linear tetrahedral elements with 241,845 nodes. Of these, the skull consists of 43,614 elements and 14,591 nodes, the skin of 33,821 elements and 11,250 nodes, the cerebral gray matter of 666,570 elements and 99,124 nodes, the cerebral white matter of 338,346 elements and 53,719 nodes, the cerebellum of 10,208 elements and 3,466 nodes, and the cerebrospinal fluid of 185,249 elements and 59,595 nodes. 

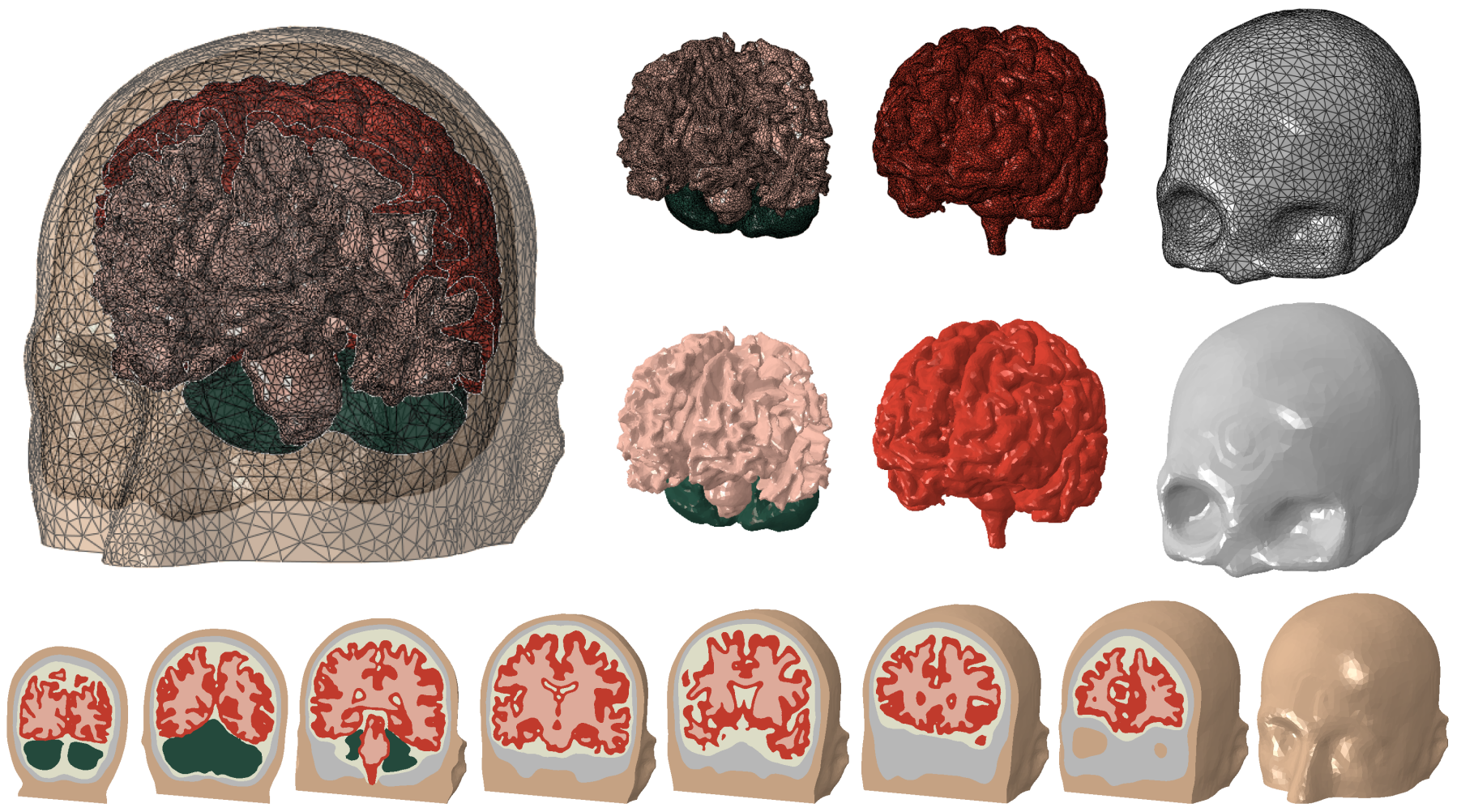

Figure 2: Personalized finite element model of the head including the skull (gray), the skin (brown), the cortical gray matter (red), the inner white matter (pink), the cerebellum (green), and the cerebrospinal fluid (beige). The top left image shows the specific relative locations of these individual substructures and their dimensions within the skull. The six images on the top right visualize mesh contours and the geometric outlines of cerebral and cerebellar white matter, the cerebral gray matter, and the skull. The images in the lower row show coronal sections across the entire brain model.

Table 2: Personalized finite element model of the head with individual substructures and number of elements and nodes.

\begin{tabular}{|l|c|c|c|}
\hline substructure & number of elements & number of nodes & element type \\
\hline \hline skull & 43,614 & 14,591 & C3D4 \\
skin & 33,821 & 11,250 & C3D4 \\
\hline \hline cerebral gray matter & 664,570 & 99,124 & C3D4H \\
cerebral white matter & 338,346 & 53,719 & C3D4H \\
cerebellum & 10,208 & 3,466 & C3D4H \\
cerebrospinal fluid & 185,249 & 59,695 & C3D4H \\
\hline \hline whole head model & 1,275808 & 241,845 & C3D4/C3D4H \\
\hline
\end{tabular}

C3D4 linear tetrahedral element, C3D4H hybrid linear tetrahedral element.

Table 2 summarizes our personalized brain model with the individual substructures, the number of elements, and the number of nodes. We import our brain model into the commercial finite element software package Abaqus (Dassault Systèmes Simulia Corp., Providence, RI, USA) where we prescribe the material models, the boundary conditions, and the interaction constraints for the individual substructures.

\subsection{Decompressive craniectomy model}

We use the brain model to simulate the effects of a unilateral decompressive craniectomy. Our finite element model provides a personalized, anatomically detailed representation of the head to investigate the mechanical strains and radial and tangential stretches upon brain tissue swelling as a potential source of diffuse axonal injury. We simulate the surgical intervention of skull opening and allow the brain to bulge outward to reduce the intracranial pressure. In the clinical setting, a decompressive craniectomy usually takes four to six hours [33]. The actual opening of the skull involves the successive creation and removal of the bone flap and the gradual opening of the dura, which takes approximately half an hour [41]. This suggests that we can neglect effects of acceleration and simulate the decompressive craniectomy as a quasi-static procedure. On these time scales, we also neglect porous 
and viscous effects, which typically decay within three to five minutes [6], and simulate brain tissue as purely hyperelastic [43]. The challenge of this procedure is to identify an optimal opening location and an optimal opening size to maximize the control of the elevated intracranial pressure and, at the same time, minimize the risk of axonal damage.
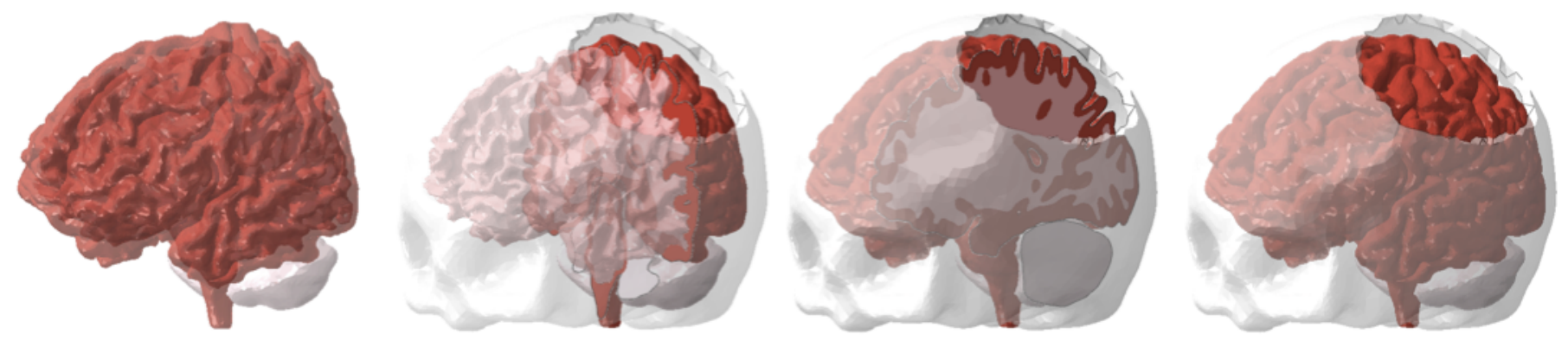

Figure 3: Personalized decompressive craniectomy model. We remove 2,494 skull elements from the initial whole head model to introduce a circular opening with a diameter of $10 \mathrm{~cm}$ in the left posterior skull. This allows the swelling brain to bulge outward and release the elevated intracranial pressure. Whole brain without skull (left), decompressive craniectomy model with frontal and lateral brain regions removed for visualization purposes (middle), and decompressive craniectomy model of whole brain with surgically opened skull (right).

Figure 3 illustrates our decompressive craniotomy model, which we create from the whole head model by introducing a circular opening with a diameter of $10 \mathrm{~cm}$ in the left posterior skull. To generate the opening, we semi-manually remove a total of 2,494 skull elements. Upon swelling, the brain can now bulge outward and release the elevated intracranial pressure. Figure 3 shows the whole brain without skull, the decompressive craniectomy model with frontal and lateral brain regions removed for visualization purposes, and the decompressive craniectomy model of the whole brain with the surgically opened skull.

Figure 4 shows the boundary and loading conditions of our decompressive craniectomy model. To limit the motion of the inferior soft tissue regions, we apply homogeneous Dirichlet boundary conditions at the lower outer surface of the cerebrospinal fluid shown in red [14]. To reduce the computational time, we ignore the skin layer and model the skull as a rigid body. We assume a tight contact between all substructures, i.e., between the cerebral gray and white matter, the cerebellum, and the cerebrospinal fluid [14]. Only at the interface between the cerebrospinal fluid and the skull, we apply frictionless contact to enable a free sliding of the brain along the skull shown in pink. We simulate the swelling of the brain by prescribing a local volumetric expansion in a predefined white matter region. To explore the effects of decompressive craniectomy, we investigate three different scenarios of brain swelling: a maximum volumetric expansion of $10 \%$ in the white matter tissue of both hemispheres, exclusively in the collateral left hemisphere, and exclusively in the contralateral right hemisphere. In all three scenarios, the circular skull opening is located in the posterior left hemisphere of the brain. We quantify and compare the mechanical response in terms of the overall deformation field, local stretches and strains, and the midline shift. The midline shift is a common clinical indicator to characterize the degree of subcortical swelling and axonal damage.
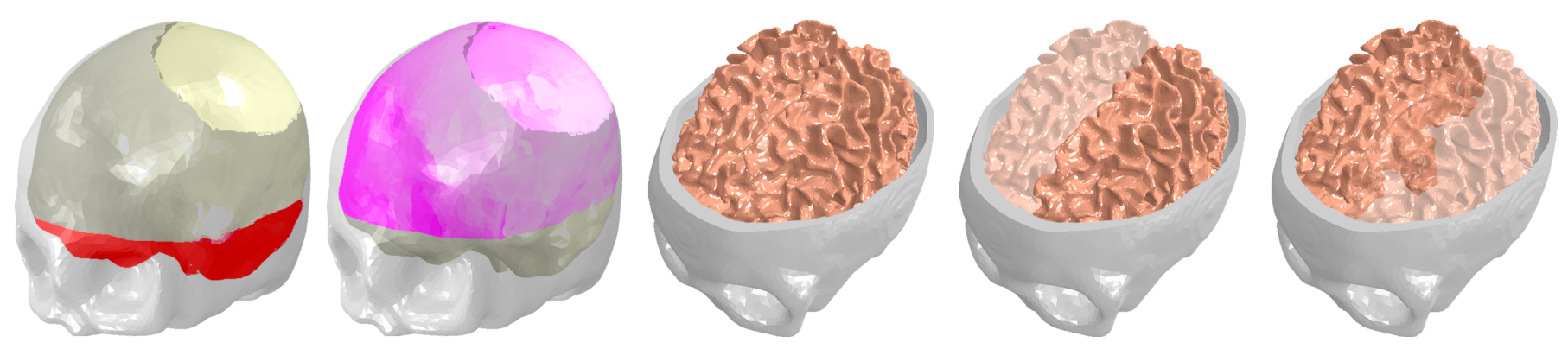

Figure 4: Boundary and loading conditions of our decompressive craniectomy model. Homogeneous Dirichlet boundary conditions at the lower outer surface of the cerebrospinal fluid (red region) limit the motion of the inferior soft tissue regions (left). Frictionless contact at the remaining skull-fluid interface (pink region) enables a free sliding of the brain along the skull (second from left). Prescribed expansion mimics brain swelling. We compare the effects of decompressive craniectomy for three distinct cases: swelling of the white matter tissue in both hemispheres (middle), exclusively in the left hemisphere (second from right), and exclusively in the right hemisphere (right). 

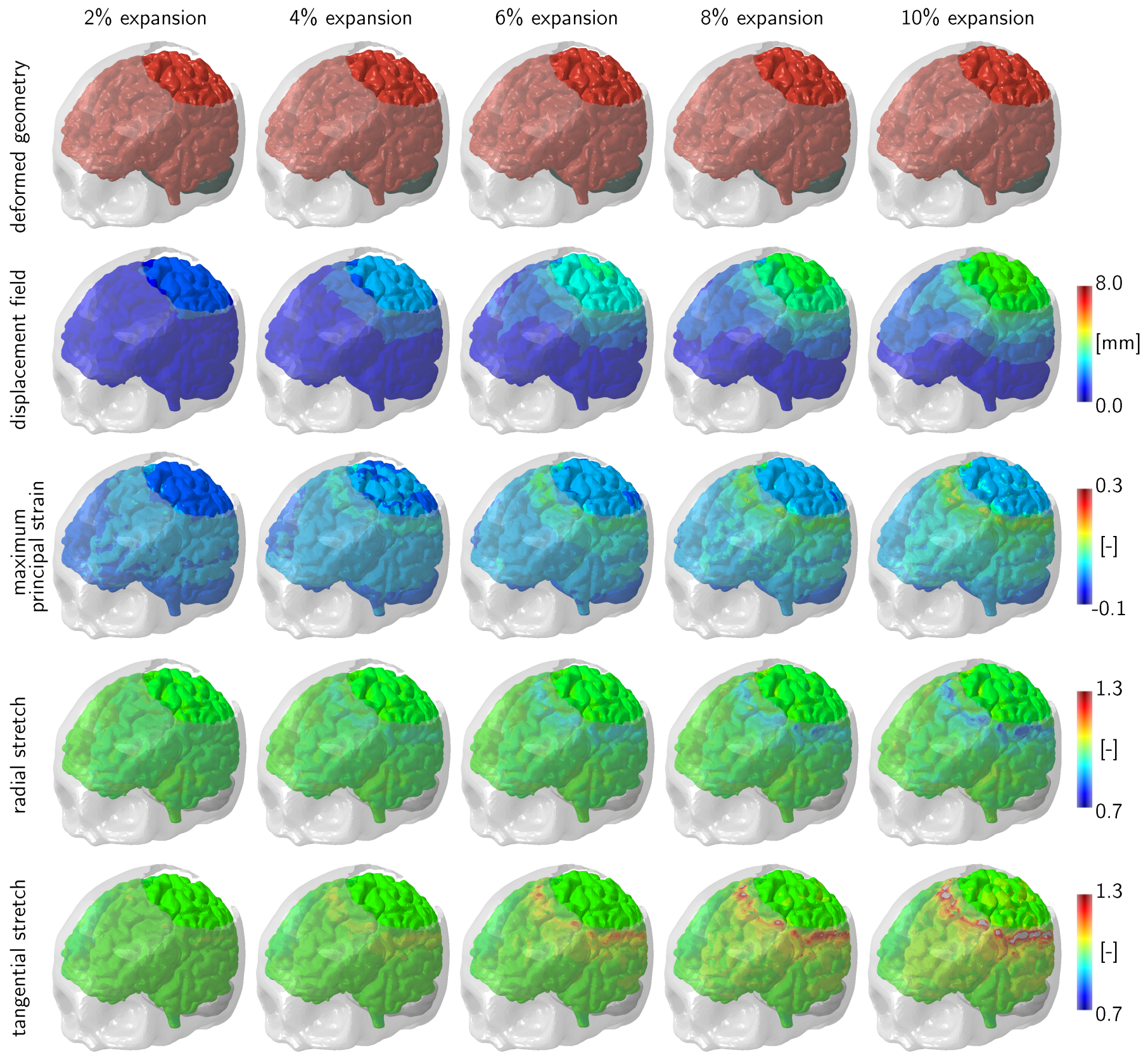

Figure 5: Timeline of progressive swelling of the left hemisphere. Columns illustrate the time points at 2\%, $4 \%$, $6 \%, 8 \%$, and $10 \%$ volumetric expansion in the white matter tissue of the left hemisphere; rows show the deformed geometry, displacement, maximum principal strain, radial stretch, and tangential stretch. Strain and stretch are largest along the edge of the skull opening suggesting that axons in this region are most vulnerable to damage and diffuse axonal injury.

\section{Results}

\subsection{Progressive unilateral brain swelling}

Figure 5 shows the evolution of progressive unilateral swelling of the left hemisphere. The individual columns are associated with the time points at $2 \%, 4 \%, 6 \%, 8 \%$, and $10 \%$ volume expansion of the left white matter tissue. The individual rows show the deformed geometry, displacement, maximum principal strain, radial stretch, and tangential stretch. The displacement field illustrates the gradual outward herniation of the gray and white matter tissue resulting in a maximum displacement on the order of $6 \mathrm{~mm}$. Strains and stretches take extreme values along the edge of the skull opening. These high values suggest that axons in this region are most vulnerable to damage and diffuse axonal injury. The maximum principal strain field shows a notable asymmetry: The average maximum principal strain of 5\% in the swelling left hemisphere is twice as large as the strain in the non-swelling right hemisphere, which is squeezed against the outer right skull. Inside the brain, the average axonal stretch differs by about 
$10 \%$ between both hemispheres in accordance with the prescribed amount of swelling. Axons in the swelling left hemisphere are stretched more than axons in the non-swelling right hemisphere. Along the edge of the skull opening increased swelling causes a severe compression in the contact region. In the gray matter tissue, this compression of up to $24 \%$ could trigger severe damage of dendritic connections in the cortex and cause axonal injury in the white matter layer underneath. Simultaneously, we observe shearing of up to $28 \%$ in the gray and white matter tissue. These high shear strains in both tissue types could trigger axonal damage and lead to cortical and subcortical ischemic cell death due to restricted blood flow, restricted oxygen supply, and fiber rupture.

deformed geometry
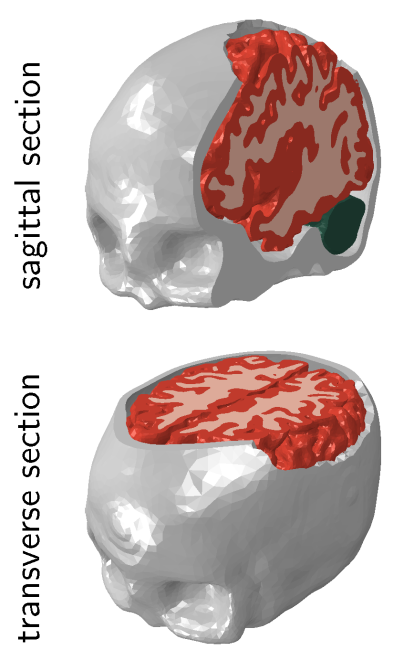

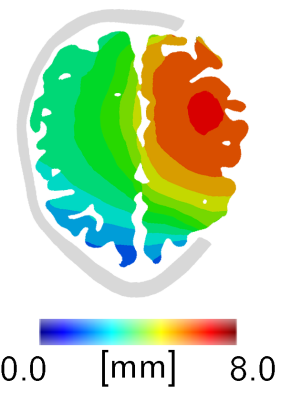

displacement field

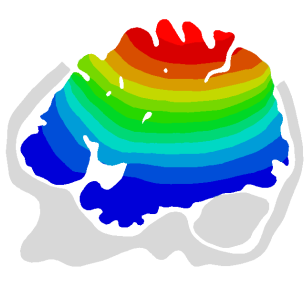

$0.0 \quad[\mathrm{~mm}] \quad 8.0$
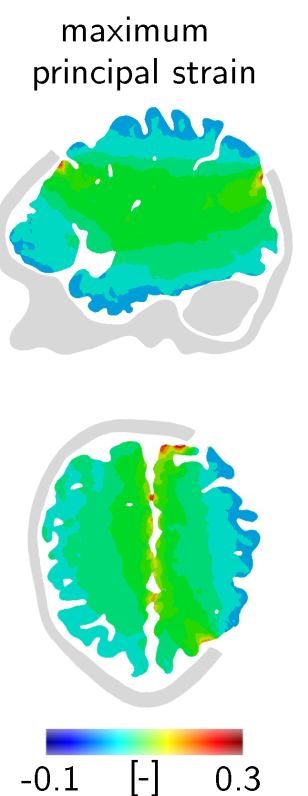

radial stretch
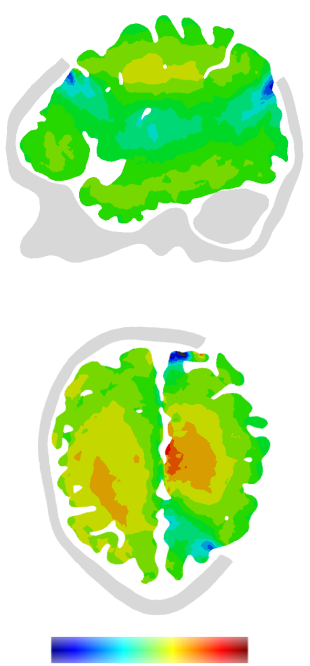

$0.7 \quad[-] \quad 1.3$ tangential stretch
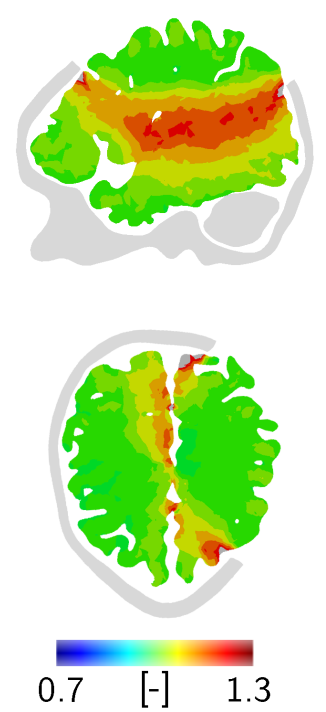

Figure 6: Sagittal and transverse sections of the computational simulation of decompressive craniectomy in response to swelling of the left hemisphere. Columns show the sagittal and transverse sections of the deformed geometry, displacement, maximum principal strain, radial stretch, and tangential stretch.

Figure 6 provides insight into the deformed geometry, displacement, maximum principal strain, radial stretch, and tangential stretch inside the brain. Inspired by the magnetic resonance images in Figure 1, which provide sagittal, coronal, and transverse sections of the brain, we visualize the mechanical behavior inside the brain in response to swelling of the left hemisphere in representative sagittal and transverse sections. Especially transverse sections are widely used in medial diagnostics to quantify the state of the swollen brain since they clearly visualize the in-plane deformation field, the dimension of the outward bulge, and the midline shift. The displacement field clearly highlights the magnitude of outward bulging on the open side of the skull. The maximum principal strain takes maximum values on the order of $30 \%$ in a wide circular region around the opening. Across the gyri of the outward bulging region, maximum principal strains are on the order of $-10 \%$ indicating a slight compression of the outer surface across the bulge. The radial stretch takes maximum values on the order of 1.3 in the center of the bulge where bulging is largest and minimum values on the order of 0.7 localized along the edge of the opening where tissue deformation is locally constrained by the skull. The tangential stretch takes maximum values of 1.3 all around the opening where outward sliding is constrained by the edge of the skull. In summary, we conclude that radial stretches are tensile inside the bulge and compressive around the skull opening, which is also the region where tangental stretches are largest.

\subsection{Sensitivity of skull opening relative to swelling site}

Figure 7 compares three different cases of brain swelling with a volumetric expansion of 10\%: swelling in the entire white matter tissue of both hemispheres; exclusively in the left hemisphere, collateral, at the site of skull opening; and exclusively in the right hemisphere, contralateral, opposite to the skull opening. Naturally, swelling the entire white matter tissue in both hemispheres causes the largest displacements, strain, and stretch. Our simulations predict maximum displacements of $9.2 \mathrm{~mm}$ for full swelling, $7.9 \mathrm{~mm}$ for swelling of the right hemisphere, and $6.9 \mathrm{~mm}$ for swelling of the left hemisphere. These results suggest that opening the skull on the side of swelling will lead to smaller tissue deformation, strain, and stretch than opening the skull on the side opposite to the origin of swelling. When opening the skull on the side of swelling, the maximum principal strain is 30\% lower in comparison to opening the skull upon swelling of both hemispheres and $16 \%$ lower in comparison to swelling of the white matter of the right hemisphere. Even more dramatically, when opening the skull on the side of swelling, the radial compressive stretch in the region around the skull opening is by a factor two lower as compared to both swelling hemispheres, and by $20 \%$ lower as compared to right hemisphere swelling. This difference suggests that axons around the opening are compressed least in a collateral craniectomy where the opening is co-located with the side of swelling. The maximum shear stretch in gray and white matter tissue is 1.48 for 

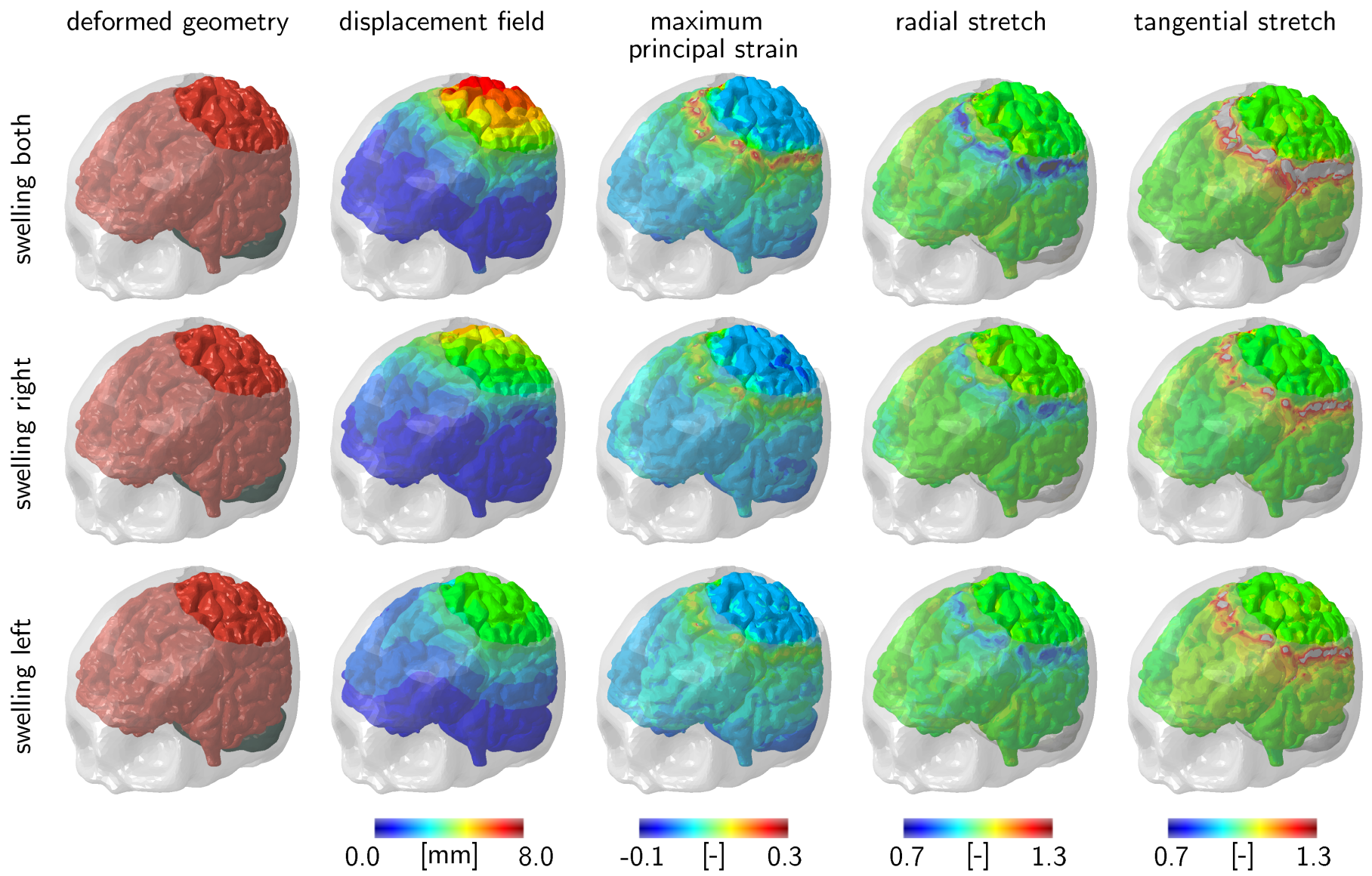

Figure 7: Mechanics of three different scenarios of brain swelling. Rows illustrate a swelling of $10 \%$ in the white matter tissue of both hemispheres, exclusively in the right hemisphere, and exclusively in the left hemisphere. Columns show the deformed geometry, displacement, maximum principal strain, radial stretch, and tangential stretch.

swelling of both hemispheres. It is $7 \%$ lower for swelling of the right hemisphere and $10 \%$ lower for swelling of the left hemisphere. For a circular skull opening and a brain deformation that decays parabolically with distance from the craniectomy edge, we can approximate the brain volume $\Delta V$ which bulges outward through the following relation, $\Delta V=\frac{2}{3} \pi r^{2} h$, where $r$ is the radius and $h$ is the height of the bulge [20]. In our three numerical examples with a radius of $r=5 \mathrm{~cm}$, this approximation predicts a bulge volume of $48 \mathrm{ml}$ for swelling of both hemispheres, $41 \mathrm{ml}$ for swelling of the right hemisphere, and $36 \mathrm{ml}$ for swelling of the left hemisphere, which is in agreement with the computationally predicted differences in displacement, strain, and stretch illustrated in Figure 7. In summary, we conclude that despite a similar fractional swelling of $10 \%$ in all three cases, we observe a markedly different mechanical response. Our simulations suggest that brain deformation, tissue strain, and axonal stretch are lowest if swelling and skull opening are co-located on the same hemisphere. This result is consistent with the procedure that is most commonly performed in clinical practice.

\subsection{Sensitivity of midline shift with respect to swelling site}

Figure 8 compares the overall displacement field and the midline shift for a swelling of $10 \%$ in the white matter tissue of both hemispheres, exclusively in the left hemisphere, and exclusively in the right hemisphere. In a side-by-side comparison, we illustrate the undeformed and deformed configurations in top view and in transverse sections. The transverse sections clearly highlight the differences between the three cases with swelling of both hemispheres causing the largest midline shift. When swelling and opening are co-located on the same side of the brain, the midline shift is $45 \%$ smaller in comparison to swelling in both hemispheres. When swelling and opening are located on opposite sides of the brain, the midline shift is only $15 \%$ smaller in comparison to swelling in both hemispheres. To visualize the midline shift and rotation of the brain, the third column of Figure 8 illustrates the overlaid undeformed and deformed configurations highlighted in blue and red. Interestingly, the right hemisphere remains almost unaffected by the collateral craniectomy where swelling and opening are co-located in the left hemisphere. For the two other cases in which the right hemisphere is swelling, we observe not only a significant midline shift, but also a larger outward herniation. This is surgically unfavorable; it exposes a larger tissue volume to lie unprotected while the skull remains open until the swelling has decreased and 

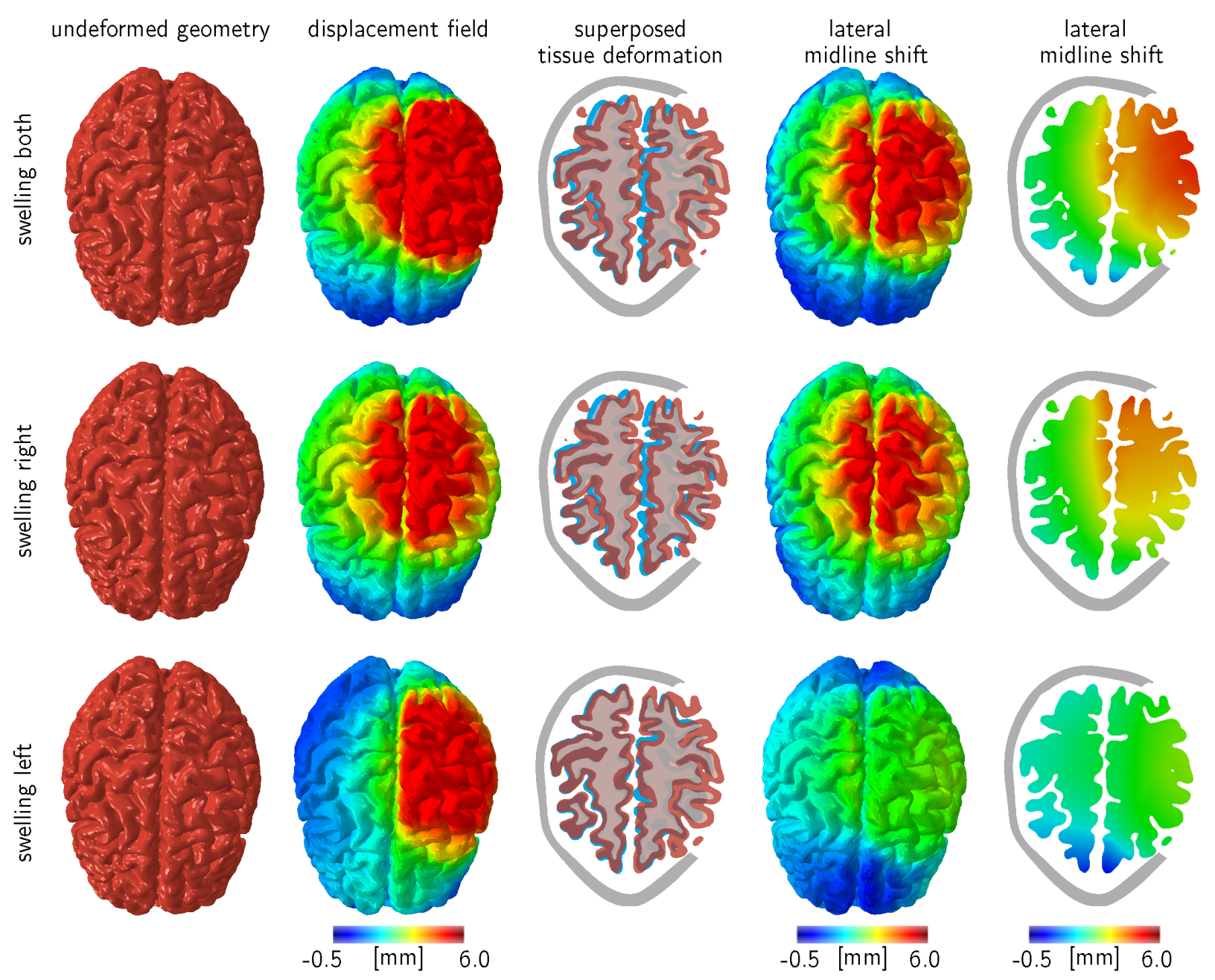

Figure 8: Midline shift for three different scenarios of brain swelling. Rows illustrate a swelling of $10 \%$ in the white matter tissue of both hemispheres, exclusively in the right hemisphere, and exclusively in the left hemisphere. Columns show the undeformed geometry, displacement field, superposed undeformed and deformed configurations in a transverse section, and the lateral midline shift from a top view and in transverse sections. The side-by-side comparison of the undeformed (blue) and deformed (red) configurations highlights not only the horizontal shift of the brain but also the rotation of both hemispheres depending on the swelling site in relation to the location of skull opening.

the intracranial pressure has returned back to its physiological value. In addition to the midline shift, we observe a rotation of the brain, where the frontal lobe is compressed within the skull while the posterior part bulges outward.

Figure 9 illustrates the lateral and superior midline shift in sagittal sections. Consistent with the results in Figure 8, we observe a similar behavior for swelling of both hemispheres and swelling of the right hemisphere shown in the first and second column. The lateral displacement in the first row is a measure of the brain pushing toward the skull opening. The lateral displacement takes maximum values at the top of the brain and gradually decreases when moving down towards the brain stem. The rotation superposed onto the translation of the brain becomes apparent in the inhomogeneous lateral displacement field. The frontal part of the brain is more restricted to move, while the posterior part of the brain can easily expand towards the skull opening. This implies that the posterior part of the brain rotates toward the skull opening while the frontal lobe is confined within the skull.

\section{Discussion}

The procedure of decompressive craniectomy has been performed for more than a century; yet, it remains controversial and ethically questionable. Computational simulations now provide insight into the mechanical consequences of the procedure with the ultimate goal to maximize stress release and minimize tissue damage. Towards this goal, the objective of this manuscript was to showcase how personalized simulations can help us to predict the dimensions of the outward bulging tissue, to understand the resulting strain field, and to identify regions at risk for local tissue damage. Specifically, we analyzed the displacement field, the maximum principal strain, the radial stretch, and the tangential stretch for a left hemicraniectomy in the case of unilateral left 


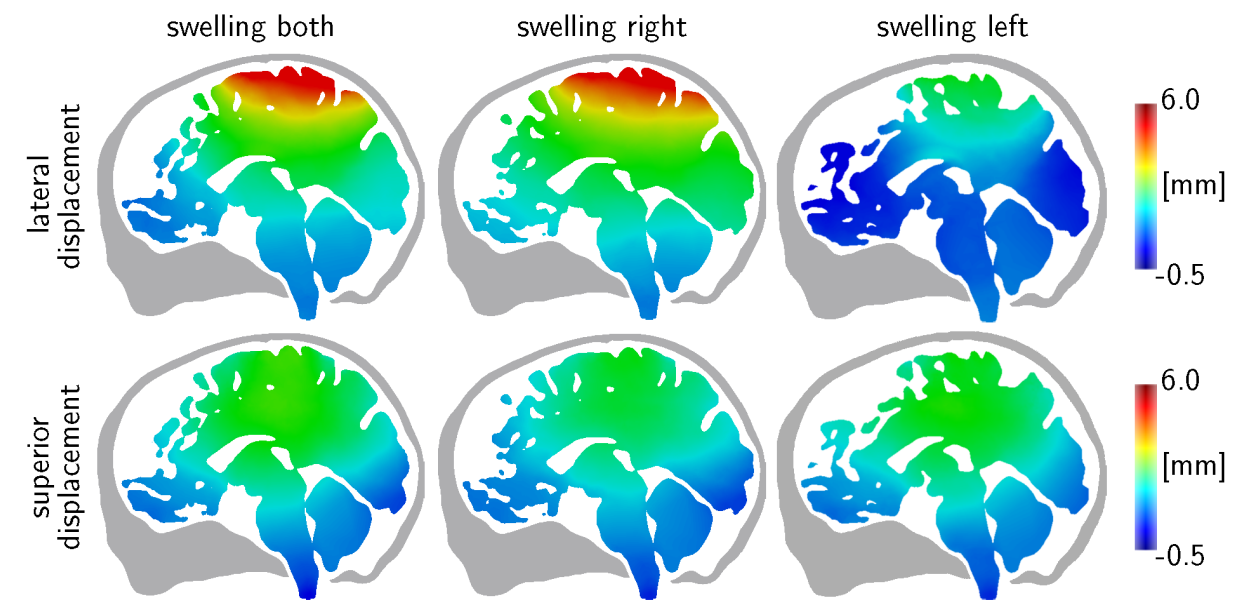

Figure 9: Midline shift for three different scenarios of brain swelling. Rows show the lateral and superior midline shift in sagittal sections. Columns illustrate a swelling of $10 \%$ in the white matter tissue of both hemispheres, exclusively in the right hemisphere, and exclusively in the left hemisphere.

swelling, unilateral right swelling, and swelling of both hemispheres. The basis of our simulation is an anatomically detailed personalized craniectomy model created from magnetic resonance images. In the absence of information from diffusion tensor imaging to characterize axon orientations, as a first approximation, we assume that all axonal fibers are oriented radially outward. This approximation allows us to interpret the radial and tangential stretches as the stretches along and perpendicular to the axons.

Our simulations revealed three potential failure mechanisms: axonal stretch, axonal compression, and axonal shear. The axonal stretch takes maximum values on the order of 1.3 in the center of the bulge where bulging is largest. The axonal stretch takes minimum values on the order of 0.7 localized along the edge of the opening where tissue deformation is locally constrained by the skull. The axonal shear takes maximum values of 1.3 all around the opening where outward sliding is constrained by the edge of the skull. While the locations of these extreme values are intuitive from a mechanics point of view, their magnitudes are unexpectedly high. Figures 5 and 7 confirm that, even for a brain swelling of only 10\%, axons are locally stretched, compressed, and sheared by up to $30 \%$. The maximum principal strain provides a more global assessment of the tissue deformation. Figures 6 and 7 show that it takes maximum values of $30 \%$ in a wide circular region around the opening and minimum values of $-10 \%$ across the outward bulging cortical surface.

Several controlled in vitro studies have revealed the mechanisms of axonal failure, however, almost exclusively under tensile loading [48]. A comprehensive series of stretch experiments with optical nerves reported damage level thresholds on the order of $18 \%$ for functional damage and $21 \%$ for morphological damage, both along the axonal direction [2]. When examining the ultrastructure of axons, it is quite intuitive that shear loading could be equally damaging to axonal function and morphology. While shear experiments are difficult to perform on single axons or axonal bundles, computational modeling can easily extract separate damage criteria for normal and tangential loading. From a failure-mechanism point of view, these criteria have been classified as volumetric-type and shear-type [10]. In fact, various continuum damage criteria have been proposed for brain tissue, either stress based or strain based, of Mises type or maximum principal type, and along or perpendicular to the axonal direction [51]. Several studies also suggest strain-rate based damage criteria, which, however, seem to be more relevant in the case of high-impact loading, e.g., during traumatic brain injury [48]. These considerations have even motivated anisotropic constitutive models, which incorporate the microstructural direction of the axonal fibers through the fourth strain invariant [7, 21], similar to the classical Holzapfel model for arterial tissue [17]. However, recent experiments with human brain tissue seem to suggest that there is no pronounced anisotropy along the axonal fiber direction [6]. This is consistent with a comprehensive review that recommends to approximate brain tissue as an isotropic hyperelastic material, but analyze its damage criteria in view of the microstructural axonal orientation [51].

In vivo measurements of strains are rare. Magnetic resonance elastography is gaining increased popularity as a technology to characterize the mechanical behavior of the human brain in vivo [11], but it has not been used to date to quantify the mechanics of decompressive craniectomy. Computed tomography images before and after a decompressive craniectomy reveal strain levels from $24 \%$ to $55 \%$, where maximum strains are located around the edge of the skull opening [22]. In combination with diffusion-weighted images to extract regional fiber orientations, this study reports average axonal strains on the order of 30\% and maximum axonal strains of 50\% localized close to the skull edge [36]. This agrees well with our computational predictions in Figures 5 and 7 , which predict localized concentrations of the maximum principal strain in the regions of skull opening. The clinically measured strain magnitudes are slightly larger because our simulations only used a local swelling of $10 \%$. Under these conditions, our predicted maximum displacements of $6 \mathrm{~mm}$ and our herniation volumes of $48 \mathrm{ml}$ for swelling of both hemispheres and $41 \mathrm{ml}$ and $36 \mathrm{ml}$ for 
unilateral swelling of the right and left hemispheres agree well with the most advanced finite element model of decompressive craniectomy, which predicted displacements of $5.2 \mathrm{~mm}$ and herniation volumes of $22 \mathrm{ml}$ [14]. Our maximum tangential stretch of 1.3 and its location around the skull opening also agree favorably with the reported maximum shear strains of 0.25 at the craniectomy edge. Although this model has a less detailed anatomic representation than ours and lacks the individual gyri and sulci, the authors report similar convergence issues beyond swelling fractions on the order of 10\% [14]. Since the simulation of sliding contact around the craniectomy edge is extremely challenging, both models fail to converge beyond a decent amount of swelling. This implies that the computationally predicted values are rather on the low end compared to clinical values. A recent study of ten hemicraniectomy patients reported herniation volumes from 20 to $140 \mathrm{ml}$, a mean herniation volume of $107.3 \mathrm{ml}$, and a mean displacement of $29.8 \mathrm{~mm}$ [13]. Concerted efforts of experimental, analytical, and computational modeling confirm though that the results scale well with the degree of swelling $[12,50]$. In agreement with a recent craniectomy study [14], our findings suggest that local regions of strain maxima are insensitive to the amount of swelling, both in location and shape. Because of their universal shape, we have introduced the term damage drops to label these regions at high risk for tissue failure [20]. As Figures 5 and 7 suggest, damage drops are always anchored at the edge of the skull opening and expand into the surrounding white matter tissue in the form of two paired drops.

From a clinical perspective, our simulations allow us to quantify the midline shift between both hemispheres and understand the motion of the ultrasoft brain within the almost rigid skull. The midline shift is a common medical indicator for the severity of intracranial swelling in stroke, cancer, and trauma. Figures 8 and 9 illustrates the midline shift for our three different clinical cases, swelling of $10 \%$ in both hemispheres, unilateral swelling of $10 \%$ in the collateral left hemisphere and in the contralateral right hemisphere. The predicted midline shift is on the order of $6 \mathrm{~mm}$ for swelling of both hemispheres and for swelling of the contralateral hemisphere. This is about twice as much as the predicted midline shift of $3 \mathrm{~mm}$ for swelling of the collateral hemisphere. Superposed to this translation, we also observe a pronounced rotation, which is naturally not reflected in the strain and stretch fields but clearly visible in Figure 8. The brain stem plays the role of an anchor at the lateral part of the brain. While the superior posterior part bulges outwards, the frontal part of the brain is constrained by the skull, which results in a rigid body rotation around the vertical axis. Again, the magnitude of rotation is larger for the contralateral craniectomy than for the collateral craniectomy. The clinical recommendation to open the skull at the side of swelling-and not at the opposite side-is rather intuitive from an engineering point of view. Interestingly, these findings disagree with a recent study, which simulated six clinical decompressive craniectomy cases and found that opening the contralateral non-injured hemisphere could potentially improve patient outcome [23]. Potential explanations for the discrepancy between those results and ours could be the role of the falx, the sequence of the simulation, and the focus on different strain components. While the discussion of collateral versus contralateral opening warrants further investigation, there seems to be a general consensus upon all studies that a unilateral craniectomy induces larger tissue strains than a bilateral craniectomy [14]. Finite element simulations could help to provide clarity and quantify the different strain fields [25]. They could also help to filter out intraoperative deformations induced by gravity, which differ for the different procedures because of the different orientations of the head [41].

Although this first prototype study already provides valuable insight into the mechanics of the brain during decompressive craniectomy, it has several limitations that we need to address in future studies: First, our model does not include a separate structure of the falx, which could play an important role in translating tissue deformation from one side of the brain to the other. Second, our model does not explicitly account for different shapes of the craniectomy edge, fillet radius or chamfer, since this seems to play a rather minor role when the dura is retained to protect the bulging brain. Third, our simulation has focused on the final state of the procedure and has not yet included dynamic strain rate effects, a viscous material behavior, or porous effects, which could play a critical role at the very instant of skull opening. Fourth, our model assumes a homogeneous brain microstructure, homogeneous connective tissue, and a homogeneous cerebrospinal fluid, which could very well display regional variations that result in heterogeneous effects. Fifth, our swelling volumes are rather low when compared to clinical studies, but we expect our general trends to remain the same, even for larger amounts of swelling. Last, and most importantly, we have only investigated the mechanical effects of skull opening. While our study can provide general guidelines, predict the overall deformation, and minimize axonal stretch and shear, it does not address risks of infection, complications of wound healing, and challenges of cranial reconstruction, all of which are important factors that need to be included in the overall process of clinical decision making.

\section{Conclusion}

Despite its long surgical tradition, decompressive craniectomy continues to be a procedure of broad criticism and ethic concern. Its surgical protocols vary widely and its technical details are mainly selected by personal preference rather than by unified standards and professional consensus. Personalized simulations can rationalize surgical decision making towards maximizing the reduction of the intracranial pressure while minimizing the damage of the surrounding tissue. In view of this goal, we created a personalized craniectomy model from magnetic resonance images and performed a virtual craniectomy by swelling regions of the brain and opening part of the skull. This allows the brain to bulge outward and release the elevated intracranial pressure. Our simulations reveal three potential failure mechanisms associated with the procedure: axonal stretch in the center of the bulge, axonal compression at the edge of the craniectomy, and axonal shear around the opening where outward sliding is locally constrained. 
Interestingly, for a swelling of only $10 \%$, axonal strain, compression, and shear reach local maxima of $30 \%$, which is beyond the reported damage thresholds for axonal tissue. In agreement with our engineering intuition, the displacement, rotation, strain, and midline shift are larger for a contralateral craniectomy, when opening the brain at the side opposite to the swelling, than for a collateral craniectomy, when opening the brain at the side of swelling, which is the preferred clinical practice. Taken together, our decompressive craniectomy model can help quantify brain deformation, tissue strain, axonal stretch, and shear to identify regions at risk for tissue damage. Ultimately, when properly validated, computational modeling can help rationalize patient selection, optimize opening locations, and select opening sizes with the overall goal to reduce brain damage and improve surgical outcomes.

\section{Acknowledgements}

The authors thank Allan L. Reiss and his group for providing the MRI scans. This work was supported by the Wolfson/Royal Society Merit Award to Alain Goriely and by the National Institutes of Health grant U01 HL119578 to Ellen Kuhl.

\section{References}

[1] Abaqus 6.14. Analysis User's Manual. SIMULIA. Dassault Systèmes. 2014.

[2] A.C. Bain, D.F. Meaney, Tissue-level thresholds for axonal damage in an experimental model of central nervous system white matter injury. J. Biomech. Eng. 122 (2000) 615-622.

[3] H. van den Bedem, E. Kuhl, Tau-ism: the Yin and Yang of microtubule sliding, detachment, and rupture. Biophys J. 109 (2015) $2215-2217$.

[4] S. Budday, P. Steinmann, E. Kuhl. The role of mechanics during brain development. J. Mech. Phys. Solids. 72 (2014) 75-92.

[5] S. Budday, R. Nay, R. de Rooij, P. Steinmann, T. Wyrobek, O.C. Ovaert, E. Kuhl. Mechanical properties of gray and white matter brain tissue by indentation. J. Mech. Behavior Biomed. Mat. 46 (2015) 318-330.

[6] S. Budday, G. Sommer, C. Birkl, C. Langkammer, J. Haybäck, J. Kohnert, M. Bauer, F. Paulsen, P. Steinmann, E. Kuhl, G. Holzapfel. Mechanical characterization of human brain tissue. submitted for publication (2016).

[7] R.J.H. Cloots, J.A.W. van Dommelen, T. Nyberg, S. Kleiven, M.G.D. Geers, Micromechanics of diffuse axonal injury: influence of axonal orientation and anisotropy. Biomech. Model. Mechanobio. 10 (2011) 413-422.

[8] D.J. Cooper, J.V. Rosenfeld, L. Murray, Y.M. Arabi, A.R. Davies, P. D’Urso, T. Kossmann, J. Ponsford, I. Seppelt, P. Reilly, R. Wolfe. Decompressive craniectomy in diffuse traumatic brain injury. New Engl. J. Med. 364 (2011) 1493-1502.

[9] R.T. Cotton, C.W. Pearce, P.G. Young, N. Kota, A.C. Leung, A. Bagchi, S.M. Qidwai, Development of a geometrically accurate and adaptable finite element head model for impact simulation: the Naval Research Laboratory-Simpleware Head Model. Comp. Meth. Biomech. Biomed. Eng. 19 (2016) $101-113$.

[10] T. ElSayed, A. Mota, F. Fraternali, M. Ortiz. Biomechanics of traumatic brain injury. Comp. Meth. Appl. Mech. Eng. 197 (2008) $4692-4701$.

[11] Y. Feng, E.H. Clayton, Y. Chang, R.J. Okamoto, P.V. Bayly, Viscoelastic properties of the ferret brain measured in vivo at multiple frequencies by magnetic resonance elastography. J. Biomech. 46 (2013) 863-870.

[12] T.L. Fletcher, A.G. Kolias, P.J.A. Hutchinson, M.P.F. Sutcliffe, Development of a finite element model of decompressive craniectomy. PLoS ONE. 9 (2014) e102131.

[13] T.L. Fletcher, A.G. Kolias, P.J.A. Hutchinson, M.P.F. Sutcliffe, An improved method for assessing brain deformation after decompressive craniectomy. PLoS ONE. 9 (2014) e110408.

[14] T.L. Fletcher, A.G. Kolias, H. Adams, P.J.A. Hutchinson, M.P.F. Sutcliffe, Modelling of brain deformation after decompressive craniectomy. submitted for publication.

[15] G. Franceschini, D. Bigoni, P. Regitnig, G.A. Holzapfel, Brain tissue deforms similarly to filled elastomers and follows consolidation. J Mech Phys Solids. 54 (2006) 2592-2620.

[16] C.P. Gao, B.T. Ang. Biomechanical modeling of decompressive craniectomy in traumatic brain injury. Acta Neurochir. Suppl. 102 (2008) $279-282$.

[17] T.C. Gasser, R.W. Ogden, G.A. Holzapfel, Hyperelastic modelling of arterial layers with distributed collagen fibre orientations. J. Royal Soc. Interface 3 (2006) 1535.

[18] A. Goriely, M.G.D. Geers, G.A. Holzapfel, J. Jayamohan, A. Jerusalem, S. Sivaloganathan, W. Squier, J.A.W. van Dommelen, S. Waters, E. Kuhl, Mechanics of the brain: Perspectives, challenges, and opportunities. Biomech. Mod. Mechanobio. 14 (2015) 931-965.

[19] A. Goriely, S. Budday, E. Kuhl, Neuromechanics: from neurons to brain. Adv. Appl. Mech. 48 (2015) $79-139$.

[20] A. Goriely, J. Weickenmeier, E. Kuhl, Stress singularities in swelling soft solids. submitted for publication.

[21] M.A. Holland, K.E. Miller, E. Kuhl. Emerging brain morphologies from axonal elongation. Ann Biomed Eng. 43 (2015) $1640-1653$.

[22] H. von Holst, X. Li, S. Kleiven. Increased strain levels and water content in brain tissue after decompressive craniectomy. Acta Neurochir. 154 (2012) 1583-1593.

[23] H. von Holst, X. Li, Decompressive craniectomy (DC) at the non-injured side of the brain has the potential to improve patient outcome as measured with computational simulation. Acta Neurochir. 156 (2014) 1961-1967.

[24] G.A. Holzapfel, Nonlinear Solid Mechanics: A Continuum Approach for Engineering. John Wiley \& Sons. 2000.

[25] J. Hu, X. Jin, J.B. Lee, L. Zhang, V. Chaudhary, M. Guthikonda, K.H. Yang, A.I. King, Intraoperative brain shift predictions using a 3D inhomogeneous patient-specific finite element model. J. Neurosurg. 106 (2007) 164-169.

[26] P.J. Hutchinson, E. Corteen, M. Czosnyka, A.D. Mendelow, D.K. Menon, P. Mitchell, G. Murray, J.D. Pickard, E. Rickels, J. Sahuquillo, F. Servadei, G.M. Teasdale, I. Timofeev, A. Unterberg, P.J. Kirkpatrick, Decompressive craniectomy in traumatic brain injury: the randomized multicenter RESCUEicp study. Acta Neurochir Suppl. 2006;96:17-20.

[27] P. Hutchinson, I. Timofeev, P. Kirkpatrick, Surgery for brain edema. Neurosurg Focus. 22 (2007):E14.

[28] H.R. Jones, T.M. Burns, M.J. Aminoff, DSc, S.L. Pomeroy, Netter Collection of Medical Illustrations: Nervous System: Part I, Brain, Volume 7, 2013, Saunders Elsevier, Philadelphia.

[29] S. Kleiven, H. von Holst, Consequences of head size following trauma to the human head. J. Biomech. 35 (2002) 153-160.

[30] S. Kleiven, Evaluation of head injury criteria using a finite element model validated against experiments on localized brain motion, intracerebral acceleration, and intracranial pressure. Int. J. Crashworthiness. 11 (2006) 65-79.

[31] T. Kocher. Hirnerschütterung, Hirndruck und Chirurgische Eingriffe bei Hirnkrankheiten. Alfred Hölder, Wien 1901.

[32] A.G. Kolias, P.J. Kirkpatrick, P. Hutchinson. Decompressive craniectomy: past, present and future. Nature Rev. Neurol. 9 (2013) $405-415$. 
[33] R. Komotar, C. Kellner, M. Otten, S. Connolly, G. Mc Khann. Bilateral frontotemporal decompressive craniectomy (Kjellberg Procedure). Am Assoc Neurolog Surg Neurosurg (2011) https://www.youtube.com/watch?v=sEqkejfG9Ys.

[34] G. Lang, P.S. Stewart, D. Vella, S.L. Waters, A. Goriely, Is the Donnan effect sufficient to explain swelling in brain tissue slices? J. Royal Soc. Interface. 11 (2014) 20140123.

[35] E. Lejeune, A. Javili, J. Weickenmeier, E. Kuhl, C. Linder, Tri-layer wrinkling as a mechanism for anchoring center initiation in the developing cerebellum. Soft Matter. 12 (2016) 5613-5620

[36] X. Li, H. van Holst, S. Kleiven, Decompressive craniectomy causes significant strain increase in axonal fiber tracts. J. Clinical Neurosci. 20 (2013) 509-513.

[37] L.A. Mihai, L.K. Chin, P.A. Janmey, A. Goriely, A hyperelastic constitutive model for compression stiffening applicable to brain and fat tissues. J. Royal Soc. Interface. 12 (2015) 20150486.

[38] M. Mooney. A theory of large elastic deformation. J. Appl. Phys. 11 (1940) 582-592.

[39] R.W. Ogden. Large deformation isotropic elasticity - On the correlation of theory and experiment for incompressible rubberlike solids. Proc. Roy. Soc. London A. $326(1972) 565584$.

[40] C.C. Ploch, C.S. Mansi, J. Jayamohan, E. Kuhl. Using 3D printing to create personalized brain models for neurosurgical training and preoperative planning. World Neurosurg. 90 (2016) 668-674.

[41] T.M. Quinn, J.J. Taylor, J.A. Magarik, E. Vought, M.S. Kindy, D.B. Ellegala, Decompressive craniectomy: technical note Acta Neurol. Scandinav. 123 (2011) 239-244.

[42] R.S. Rivlin. Large elastic deformations of isotropic materials. IV. Further developments of the general theory. Phil. Trans. Roy. Soc. London. 241 (1948) 379-397.

[43] R. de Rooij, E. Kuhl E. Constitutive modeling of brain tissue: current perspectives. Appl Mech Rev. 68 (2016) 010801.

[44] M. Saggar, E.M.Quintin, E. Kienitz, N.T. Bott, Z. Sun, W.C. Hong, Y. Chien, N. Liu, R.F. Dougherty, A. Royalty, G. Hawthorne, A.L. Reiss, Pictionary-based fMRI paradigm to study the neural correlates of spontaneous improvisation and figural creativity, Scientific Reports. 5 (2015) 10894.

[45] J. Sahuquillo, F. Martinez-Ricarte, M.A. Poca, Decompressive carniectomy in traumatic brain injury after the DECRA trial. Where do we stand? Curr. Opin. Crit. Care. 19 (2013) 101-106.

[46] S.I. Stiver, Complications of decompressive craniectomy for traumatic brain injury. Neurosurg. Focus. 26 (2009) E7.

[47] F. Tagliaferri, G. Zani, C. Iaccarino, S. Ferro, L. Ridolfi, N. Basaglia, P. Hutchinson, F. Servadei, Decompressive craniotomies, facts and fiction: a retrospective analysis of 526 cases. Acta Neurochir. 154 (2012) 919-916.

[48] M.D. Tang-Schomer, A.R. Patel, P.W. Baas, D.H. Smith, Mechanical breaking of microtubules in axons during dynamic stretch injury underlies delayed elasticity, microtubule disassembly, and axon degeneration. FASEB Journal. 24 (2010) 1401-1410.

[49] J. Weickenmeier, R. de Rooij, S. Budday, P. Steinmann; T.C. Ovaert, E. Kuhl, Brain stiffness scales linearly with myelin content. Acta Biomat. available online first, doi: 10.1016/j.actbio.2016.07.040.

[50] J. Weickenmeier, E. Kuhl, A. Goriely, The mechanics of decompressive craniectomy: Bulging in idealized geometries. submitted for publication.

[51] R.M. Wright, K.T. Ramesh, An axonal strain injury criterion for traumatic brain injury. Biomech. Model. Mechanobio. 11 (2011) $245-260$.

[52] P.G. Young, T.B.H. Beresford-West, S.R.L. Coward, B. Notarberardino, B. Walker, A. Abdul-Aziz, An efficient approach to converting 3D image data into highly accurate computational models, Phil. Trans. Royal Soc. A. 366 (2008) 3155-3173. 